$\mathrm{Ci}$ indeed binds these sites in vivo. Hh signalling therefore seems to promote $S$ phase by direct induction of Cyclin E expression, as well as Cyclin D.

This study shows a direct link between $\mathrm{Hh}$ signalling and cell growth (through Cyclin D) and proliferation (through both Cyclin D and Cyclin E). And, as the authors conclude, "constitutive Hh signalling, which promotes deregulated expression of G1-S cyclins that have been associated with diverse forms of human cancer, would promote both cell proliferation and growth in tumours".

Alison Mitchell Editor, Nature Reviews Molecular Cell Biology

D) References and links ORIGINAL RESEARCH PAPER DumanScheel, M., Weng, L. \& Du, W. Hedgehog regulates cell growth and proliferation by inducing Cyclin D and Cyclin E. Nature 417 299-304 (2002)

\section{WEB SITE}

Wei Du's lab:

http://devbio.bsd.uchicago.edu/index3.html?co ntent=faculty/wDu/index.html

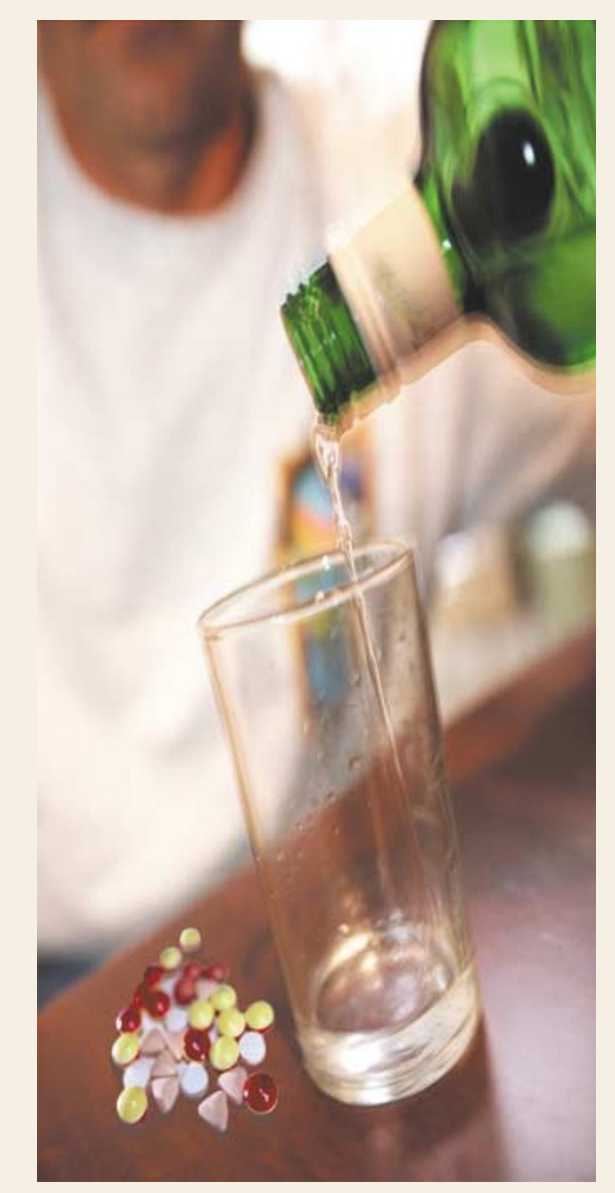

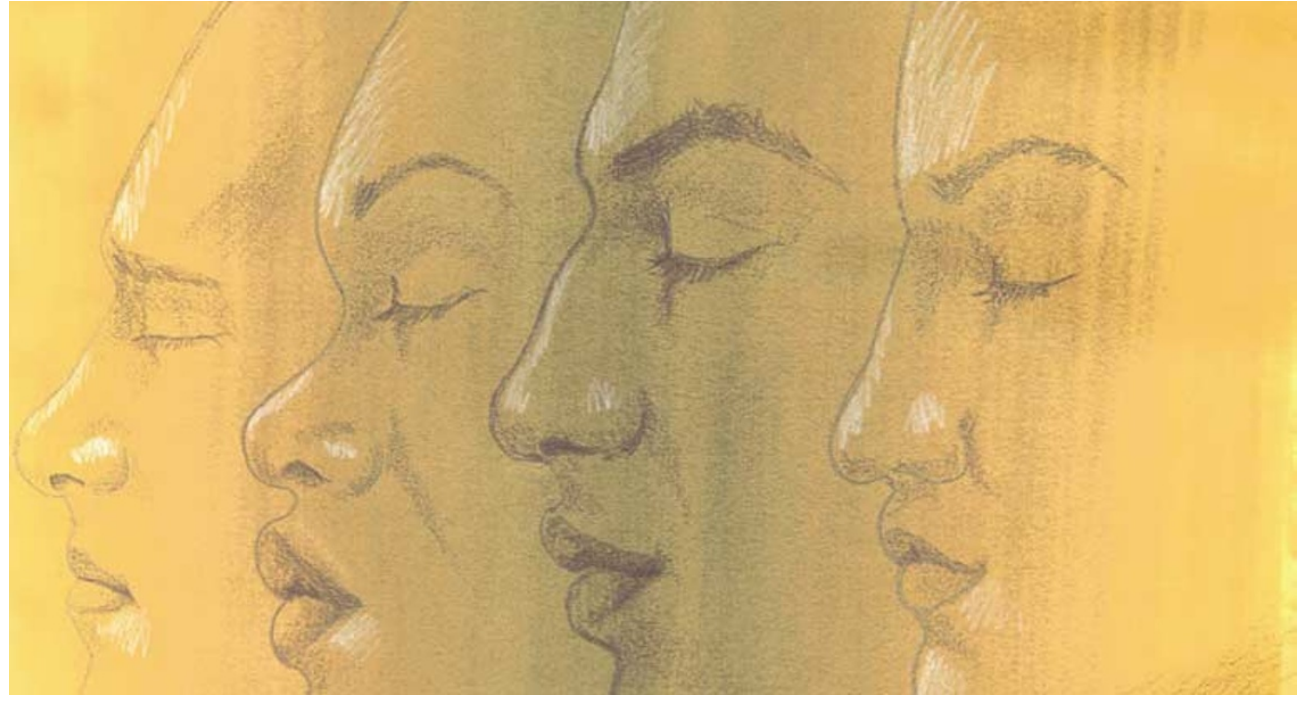

ONCOGENESIS

\title{
The many faces of MYC
}

The oncogene c-MYC is known to stimulate both cell life and cell death - two opposing processes that battle for supremacy in c-MYC-induced tumours. But now, two groups have reported in Molecular Cell that c-MYC can also induce the accumulation of reactive oxygen species (ROS), with very different results.

Omid Vafa et al. were interested in the finding that expression of c-MYC could induce chromosomal abnormalities. This could be a direct effect, or an indirect effect of c-MYC's ability to drive cells into $S$ phase prematurely, and their aim was to distinguish between these possibilities.

They developed an in situ TUNEL-based assay to allow them to visualize damaged DNA, and used this to confirm that c-MYC activation - achieved using a tamoxifen-inducible system - in normal human fibroblasts did indeed cause DNA damage. Cell-cycle analysis showed that only $1 \%$ of cells had entered $S$ phase 8-9 hours after c-MYC induction, but that most cells had an average of 23 TUNEL foci by 4 hours, which increased to $\sim 70$ /cell after 8-9 hours.

So, c-MYC expression can cause DNA damage independently of cell cycling; could the mechanism be a product of c-MYC's apoptotic programme? This possibility was ruled out because apoptotic markers - such as cytochrome $c$ release - were not seen, and addition of a caspase inhibitor did not affect the number of TUNEL foci.

Instead, c-MYC seems to induce accumulation of the metabolic intermediate ROS - which can damage DNA directly or by activating topoisomerases - 3-4 hours after c-MYC activation. Treating cells with antioxidants prevents ROS accumulation, and hence DNA damage. c-MYC-expressing cells also show decreased viability, as cells arrest in a senescence-like state, but this is also mitigated by antioxidants.

Interestingly, although c-MYC induces growth arrest as a result of DNA damage, it also seems to partly overcome the p53-induced growth arrest. Cells that are treated with $\gamma$-irradiation normally block in G1 — only $1.2 \%$ had entered S phase after 24 hours - but c-MYC activation resulted in $11.5 \%$ entering $S$ phase at the same time point.

So, c-MYC induces accumulation of ROSwhich damages DNA — and also impairs the arrest response, which could further increase genomic instability to provide a growth advantage for cancer cells.

However, Hirokazu Tanaka et al. obtained different results. They also showed that expression of c-MYC - in NIH-3T3 and Saos-2 cell lines following serum deprivation induced ROS, but that instead of causing DNA damage and growth arrest, it induced apoptosis. The mechanism behind the accumulation of ROS seems to be that c-MYC induces E2F1, which inhibits the transcription factor NF- $\kappa B$, thereby preventing it from transcriptionally activating the antioxidant MnSOD - hence, the net effect is an increase in ROS. But how can the discrepancy between the two effects of accumulated ROS be explained? The most obvious explanation is related to the different cell types that are used. Saos- 2 cells, for example, do not have $\mathrm{p} 53$, which could alter the response, and Omid Vafa et al. showed that rat cells expressing c-Myc underwent apoptosis, whereas normal human fibroblasts did not.

The important issue that now remains to be determined is whether c-MYC-induced ROS accumulation occurs in human cells in vivo to promote tumorigenesis.

Emma Greenwood

\section{(9) References and links}

ORIGINAL RESEARCH PAPERS $V a f a, O$. et al. c-Myc can induce DNA damage, increase reactive oxygen species, and mitigate p53 function: a mechanism for oncogene-induced genetic instability. Mol. Cell 9, 1031-1044 (2002) | Tanaka, H. et al. E2F1 and c-Myc potentiate apoptosis through inhibition of NF- $\mathrm{KB}$ activity that facilitates MnSODmediated ROS elimination. Mol. Cel/ 9, 1017-1029 (2002) WEB SITE

WEB SITE
Geoffrey Wahl's lab: http://www.salk.edu/faculty/wahl.html 